\title{
BIBLIOGRAPHIE
}

[1] C. C. Thiel, P. A. Clough, L. F. L. ClegG, D. N. Akam, M. Gruber, et E. Hrrons. Immersion eleaning of milking equipment. J. Dairy Res., 22, $156-165,1955$.

[2] K. E. Thоме et H. Leesment. Cleaning and desinfection of milking machines. A comparison between three different methods. Svenska. Mejeriern. Riksför. Meddel. (52), 1959.

[3] D. S. M. Phillips. Circulation washing of milking machines. N. Z. J. Agr., 17, 537-550, 1958.

[4] E. R. Gardner et N. J. BerRidGe. The deterioration of milking rubbers, II. The effect of fat. J. Dairy Res., 19, 31-38, 1952.

[5] J. C. White et G. R. Folds. A study of milking machine inflations made of neoprene compared with those of natural rubber. J. Milk and Food Technol., 17, 256-260, 1954.

[6] J. M. Jensen. Fat extraction from milker rubber with lye solutions. J. Dairy Sci., 38, 835-842, 1955.

[7] W. G. Whittlestone. Care of milking machine rubber ware. $N . Z$. J. Agric., 92, 279-284, 1956.

[8] J. M. Jensen. Dry versus lye-solution storage of milking machine rubber inflations. J. Dairy Sci., 42, 1726-1730, 1959.

[9] T. J. Claydon. Methods for studying factors that influence the sanitary condition of milking machine teat-cup liners. J. Dairy Sci., 36, 391-401, 1953.

[10] J. W. Egdell, S. B. Thomas, L. F. L. Clegg et W. A. Cuthiert. Thermoduric organisms in milk. III. Provisional standard technique for the laboratory pasteurization test for milk, and rinses and swabs of dairy equipment, with suggestions for interpretation of results. Proc. Soc. Appl. Bact., 13, 132-134, 1950.

[11] J. Richard et J. E. Auclair. Mesure de l'efficacité du nettoyage des machines à traire. XVIe Congrès Intern. de Laiterie, Copenhague. 1962 (sous presse).

\section{MÉTHODES DE L'ÉPREUVE DU DISQUE POUR DÉCELER LA PÉNICILLINE DANS LE LAIT (1)} par

\author{
Dr Lloyd D. Witter (2)
}

La méthode de l'épreuve du disque pour la détermination de la pénicilline résulte de la modification et de la simplification de la méthode du récipient d'Oxford (Oxford cup method) publiée pour

(1) Travail présenté au 53e Congrès annuel de la "Milk Industry Foundation * à Chicago, le 3 novembre 1960. (Le Québec laitier, 1960, 19, 20.)

(2) Préposé au département de la technologie alimentaire, Université d'Illinois, Urbana, Illinois. 
la première fois en 1941 [1]. Vincent et Vincent en 1944 [2] furent les premiers à concevoir et à publier le remplacement du récipient par des disques en papier, bien que Foster et WoodruFF [5] avaient déjà fait mention de leur travail. Les premières discussions des facteurs en question dans la méthode du récipient d'Oxford [5] [16] et la méthode de l'épreuve du disque [14] suggérèrent plusieurs des modifications subséquentes. Grâce à la précieuse collaboration des Laboratoires Difco, Silverman et Kosikowski [18] en vinrent à publier la première adaptation de l'épreuve du disque à l'industrie laitière. Malgré l'élaboration de beaucoup d'autres épreuves pour antibiotiques, attrayantes, plus sensibles et plus rapides, l'épreuve du disque est restée la méthode de tout repos, du pour une bonne part à sa simplicité.

\section{LE PROGÉDÉ DE BASE}

On inocule un micro-organisme sensible à la pénicilline dans un milieu de gélose fondu qui en favorisera une croissance rapide. On verse la gélose inoculée dans une boîte de Pétri et on la laisse se solidifier. On humecte un disque de papier-filtre avec l'échantillon de lait à l'essai et on le place fermement à la surface de la gélose durcie. On fait incuber la boîte de Pétri, ainsi préparé, à une température appropriée jusqu'à ce qu'on distingue sur le plat le développement du micro-organisme. Si l'échantillon de lait contient de la pénicilline (ou tout autre inhibiteur) il y aura autour du disque une zone claire dépourvue de développement microbien. Pour confirmer la présence de la pénicilline (a) on peut traiter l'échantillon de lait avec de la pénicillinase et en faire un nouvel essai (b) on peut utiliser un disque imprégné de pénicillinase pour l'essai de l'échantillon ou (c) on peut placer un disque imprégné de pénicillinase tout près du disque ordinaire qui contient l'échantillon. La présence de la pénicilline se trouve confirmée, si on parvient avec n'importe quel de ces procédés à éliminer la zone d'inhibition par l'action de la pénicillinase.

\section{VARIATIONS DU PROGÉDÉ DE BASE}

\section{Choix du micro-organisme}

Un micro-organisme approprié (a) doit évidemment être sensible à de petites concentrations de pénicilline (plus petites en réalité que la limite de l'épreuve), (b) doit se développer de préférence rapidement, dans les conditions de l'épreuve et (c) offrir l'avantage d'une 
lignée qui se prépare facilement et qui convient bien à l'incubation. Il s'emploie un grand nombre de lignées dans l'épreuve du disque mais la lignée qu'on rencontre ordinairement en industrie laitière est Bacillus subtilis ATCC 6633. Bacillus subtilis est une bactérie sporulée et à cause de cette caractéristique elle est idéale pour la préparation des cultures d'inoculation. Le micro-organisme doit s'ensemencer dans un milieu de culture qui en favorise un développement rapide.

\section{Épaisseur de la couche de gélose}

Plus la couche de gélose d'une boîte est épaisse, moins l'épreuve du disque se trouve sensible et moins il y aura de zone d'inhibition pour une concentration donnée de pénicilline. C'est pourquoi pour assurer une proportion constante entre la grandeur des zones d'inhibition et la concentration de la pénicilline, on doit mesurer avec précision la quantité de gélose destinée à chaque boîte, dont le fond a une surface plane, et permettre à la gélose de se solidifier horizontalement, pour donner ainsi à toutes les boîtes une couche de gélose semblable et uniforme. Cependant si le but de l'épreuve est de déterminer dans un échantillon de lait la présence ou l'absence de la pénicilline à un niveau de concentration, qui n'est pas un défi à la sensibilité de l'épreuve, on n'a pas besoin alors d'une couche de gélose uniforme, mais on doit la préparer assez mince pour pouvoir discerner la zone d'inhibition qui correspond à cette concentration de pénicilline dans l'échantillon.

\section{Traitement et addition de l'échantillon}

Il peut y avoir dans l'échantillon de lait des substances naturelles inhibitrices qui produiront une petite zone d'inhibition (une épreuve positive trompeuse). Ces fausses zones d'inhibition peuvent s'éliminer par le chauffage du lait à environ $180^{\circ} \mathrm{F}$, pendant cinq minutes. Si l'on ne chauffe pas l'échantillon de lait avant l'épreuve, on ne devrait pas considérer comme positifs les échantillons qui montrent de petites zones d'inhibition, à moins d'en établir la confirmation. Des disques de papier-filtre s'emploient ordinairement en deux grandeurs différentes : ceux de 0,25 pouce et ceux de 0,5 pouce. Le disque de 0,5 pouce procure une sensibilité légèrement plus grande, mais son emploi rend les épreuves un peu plus dispendieuses et un peu moins avantageuses que l'emploi du disque de 0,25 pouce, puisqu'on ne peut pas placer autant de disques par boîte. 
Pour le décelage quantitatif de la pénicilline dans le lait, il est désirable d'humecter les disques avec une pipette graduée en vue d'une répartition uniforme de l'échantillon de lait, mais pour le contrôle de la qualité, il suffit d'humecter les disques en les trempant dans l'échantillon.

\section{Température et durée de l'incubation}

On devrait régler la température d'incubation de façon à favoriser un développement rapide du micro-organisme et, pour la durée de l'incubation, on la détermine en se basant sur la rapidité avec laquelle le micro-organisme produit de la turbidité sur la boîte.

Comparaison de deux épreuves du disque généralement employées.

Dans tout le pays, des usines spécialisées dans le lait-nature font actuellement l'essai du lait des producteurs pour la présence de la pénicilline. Les deux épreuves le plus généralement employées sont la méthode de Arret et Kirschbaum [3] qui est la méthode employée par l'Administration des Aliments et Drogues ainsi que la méthode Difco modifiée qu'on trouve dans "Standard Methods for the Examination of Dairy Products" [2]. On peut comparer ces deux épreuves en se référant au tableau 1. En substance, la nouvelle méthode de Arret et Kirschbaum [3] diffère de l'ancienne méthode Difeo par le fait de réfrigérer les plats avant leur emploi et par la température d'incubation à $37^{\circ} \mathrm{C}$. Il y a lieu de croire que ces changements ont pour effet de permettre la lecture de l'épreuve après $21 / 2$ heures d'incubation plutôt qu'après une période un peu plus longue comme le conseille la méthode Difco. Witrer et Tuckey [20] étudièrent les facteurs variables de ces deux épreuves et ne purent approuver Arret et Kirschbaum dans leur recommandation de maintenir les boîtes aux températures de réfrigération pendant au moins trois jours ou au plus cinq jours. De plus, ils trouvèrent qu'on obtenait des résultats aussi satisfaisants et aussi rapides à $35^{\circ} \mathrm{C}$. qu'à $37^{\circ} \mathrm{C}$. Dans leurs expériences, une durée d'incubation de 2 1/2 heures n'était pas suffisante pour permettre une lecture adéquate des résultats, bien qu'après cette période une boîte sur sept avait des zones lisibles. Johns [7] discuta les autres variations de ces deux épreuves et ne put trouver aucune raison pour préférer l'épreuve Arret et Kirschbaum à la méthode Difco modifiée. 


\section{TABLEAU I}

COMPARAISON DE LA MÉTHODE ARRET ET KIRSGHBAUM

ET LA MÉTHODE MODIFIÉE DIFGO

POUR LA DÉTERMINATION DE LA PÉNIGILLINE DANS LE LAIT

\begin{tabular}{|c|c|c|}
\hline Facteurs variables & $\begin{array}{c}\text { Méthode de Arret } \\
\text { et Kirschbaum }\end{array}$ & Méthode modifiée Difco \\
\hline Micro-organisme .... & $\begin{array}{l}\text { Bacillus subtilis } \\
\text { ATCC } 6633\end{array}$ & $\begin{array}{l}\text { Bacillus subtilis } \\
\text { ATCC } 6633\end{array}$ \\
\hline Milieu . . . . . . . . . & Milieu antibiotique $\mathbf{l}$ & $\begin{array}{l}\text { Milieu antibiotique } 1 \text { “Whey } \\
\text { Agar " D'autres milieux } \\
\text { donnant des résultats équi- } \\
\text { valents }\end{array}$ \\
\hline Quantité par boîte ... & $10 \mathrm{ml}$ & $6 \mathrm{ml}$ \\
\hline $\begin{array}{l}\text { Instructions spéciales } \\
\text { pour la manutention } \\
\text { des boîtes } \ldots \ldots \ldots\end{array}$ & $\begin{array}{l}\text { Réfrigérer les boîtes } \\
\text { pendant au moins } 3 \\
\text { jours ou au plus } 5 \\
\text { jours }\end{array}$ & Pas de réfrigération \\
\hline $\begin{array}{c}\text { Traitement de l'échan- } \\
\text { tillon de lait } \ldots . . .\end{array}$ & Aucun & $\begin{array}{l}\text { Chauffer à } 180^{\circ} \mathrm{F} \text {. pendant } 5 \\
\text { minutes et refroidir à la } \\
\text { température de la chambre } \\
\text { pour éliminer les inhibi- } \\
\text { teurs naturels }\end{array}$ \\
\hline Grandeur du disque . . & 0,25 pouce & 0,25 ou 0,5 pouce \\
\hline $\begin{array}{l}\text { Température d'incuba- } \\
\text { tion } \ldots \ldots \ldots \ldots \ldots\end{array}$ & $37^{\circ} \mathrm{C}$ & $35^{\circ} \mathrm{C}$ \\
\hline Durée d'incubation ... & 2,5 heures $\ldots \ldots \ldots \ldots$ & $\begin{array}{l}4 \text { à } 6 \text { heures ou jusqu'à ce } \\
\text { que le développement bac- } \\
\text { térien soit visible. }\end{array}$ \\
\hline Instructions spéciales. & $\begin{array}{l}\text { Couvercles en porce- } \\
\text { laine pour les boîtes } \\
\text { de Pétri }\end{array}$ & $\begin{array}{l}\text { Boîtes de Pétri à fond plat. } \\
\text { Permettre à la gélose de se } \\
\text { solidifier avec une surface } \\
\text { horizontale }\end{array}$ \\
\hline
\end{tabular}




\section{MODIFICATIONS}

Il s'est réalisé un grand nombre de modifications ingénieuses et originales à partir de la méthode fondamentale de l'épreuve du disque. Cerny et Morris [4] augmentèrent la sensibilité de l'épreuve du disque en employant deux disques de 0,5 pouce, superposés, saturés tous les deux par le même échantillon de lait. Avec cette modification du double disque, ils parvinrent facilement à déceler 0,01 unité de pénicilline par ml de lait. KosIKow Skr et Mocquot [12] purent également sans difficulté déceler des concentrations de pénicilline aussi faibles que 0,01 unité par $\mathrm{ml}$ de lait. Ces derniers remplacèrent les disques en papier en usage dans la méthode standard de l'épreuve du disque par des tablettes circulaires de lait en poudre. Toutefois par ce procédé ils sacrifièrent la simplicité de l'épreuve du disque pour un accroissement de la sensibilité, car il fallut dessécher les échantillons de lait à basse température et opérer le pressage en tablettes.

Pital et aL. [15] développèrent une méthode relativement rapide pour le décelage des antibiotiques en combinant l'épreuve du disque et la réduction de la résazurine. Shaнano et Bodami [17] modifièrent cette méthode et l'adaptèrent au décelage des antibiotiques du lait. Dans cette méthode rapide modifiée, on sort les boîtes de l'incubateur après 40 minutes, on enlève les disques sur les boîtes, qui sont alors arrosés de résazurine et on les met de nouveau à l'incubateur. La présence de l'antibiotique se décèle par un manque de réduction de la résazurine à l'endroit où se trouvait le disque sur le plat. En employant Lactobacillus bulgaricus comme microorganisme, on a pu déceler par cette méthode combinée de la résazurine et du disque 0,04-1,67 ppm de pénicilline en 1 1/2-2 1/2 heures.

Deux modifications pratiques de l'épreuve du disque, élaborées au laboratoire de Kosikowski de l'Université de Cornell, donnèrent naissance à des épreuves de routine simplifiées pour le décelage des antibiotiques du lait. Dans une de ces épreuves [1, 13] on supprime le développement des micro-organismes aérobiques en scellant hermétiquement la boîte sous vide par une feuille d'aluminium. On active le développement des micro-organismes en laissant entrer l'air. Les boîtes ainsi préparés ne demandent pas de réfrigération avant leur emploi. La seconde méthode s'appelle l'épreuve du disque à phase inverse $[9,10,11]$. Dans cette méthode, le microorganisme est ensemencé dans une boîte de gélose non-nutritive et des disques spéciaux, imprégnés de nutriments, remplacent les disques standard en papier. On active le développement du micro- 
organisme simplement en plaçant le disque spécial à la surface de la gélose et encore ici la réfrigération des boîtes avant leur emploi n'est pas nécessaire.

Igarashi et AL. [6] développèrent récemment une épreuve du disque dans laquelle on a pu déceler facilement une teneur de 0,005 unité de pénicilline par $\mathrm{ml}$ en 1 1/2 heure. Dans cette méthode on ensemença avec Bacillus stearothermophilus la gélose (Trypticaseyeast extract glucose agar) et on fit incuber les plats à $65^{\circ} \mathrm{C}$ après avoir ajouté l'échantillon de lait sur des disques de 0,5 pouce.

L'épreuve du disque pour la détection de la pénicilline du lait était la méthode de choix dans la $10^{\mathrm{e}}$ édition de "Standard Methods for the Examination of Dairy Products" publié en 1953 [2]. Il est intéressant de noter que dans la $11^{\mathrm{e}}$ édition de cette publication, qui a paru à l'automne de 1960 , le procédé recommandé est encore la méthode fondamentale du disque. Ce sont sa sûreté et sa simplicité qui lui méritent ce privilège.

\section{RÉFÉRENCES}

[1] E. P. Abraham, E. Chain, C. M. Fletcher, H. W. Florey, A. D. Gardner, N. G. Heatley and M. A. Jennings. Further observations on penicillin. Lancet 241, 177. 1941.

[2] American Public Health Association. Standard methods for the examination of dairy products, 10th ed. New York, N. Y. 1953.

[3] B. Arret and A. Kirschbaum. A rapid dise assay method for detecting penicillin in milk. J. Milk and Food Technol., 22, 329, 1959.

[4] J. CERny and R. L. Morris. A modified dise assay method for detecting antibiotics in milk. J. Milk and Food Technol., 18, 281. 1955.

[5] J. W. Foster and H. B. Woonruff. Microbiological aspect of penicillin. I. Methods of assay. J. Bacteriol., 46, 187, 1943 ,

[6] R. T. Igarashi, R. W. Gaughman, F. E. Neldon et P. A. Hartman. Rapid antibiotic assay methods using Bacillus stearothermophilus. J. Dairy Sci., 43, 841. 1960.

[7] C. K. JoHns. Comments on a new test for penicillin in milk. J. Milk and Food Technol., 23, 6. 1960.

[8] F. V. Kosikowski. Controlling growth of test bacteria for antibiotic assays through anaerobiosis. Science, 126, 844. 1957.

[9] F. V. Kosıkowski. Field test kits for penicillin in milk. A Milk Rev., 22 (7), 38. 1960.

[10] F. V. Kosikowski et R. A. Ledford. A reverse-phase disc assay test for antibiotics in milk. J. Am. Vet. Med. Assoc., 136 (7), 297. 1960 (in Le Lait, 40, 620, 1960.

[11] F. V. Kosikowski et R. A. Ledford. Problems of design in simple field tests for antibiotics in milk. J. Dairy Sci., 43, 842, 1960. 
[12] F. V. Kostkowski et G. Mocquot. A technique to increase sensitivity of the disc assay method for antibiotics in milk. Proc. XIVth Internatl. Dairy Cong., 3 (2) : 203, 1956.

[13] R. A. LEDFORd et F. V. KosIkowski. Anaerobic studies with antibioticsensitive bacteria. J. Dairy Sci., 43, 851. 1960.

[14] Y. H. Loo, P. S. Skell, H. H. Thornberry, J. EhrLide, J. M. McGuire, G. M. Savage and J. C. Sylvester. Assay of streptomycin by the paper-dise plate method. J. Bacteriol., 50, 701. 1945.

[15] A. Pital, D. T. Disque and J. M. Lieise. A new rapid plate method for determining antibiotic sensitivity. Antibiotics and Chemotherapy. 6, 351. 1956 .

[16] W. H. Schmid et A. J. Moyer. Penicillin. I. Methods of assay. J. Bacteriol. 47, 424, 1944.

[17] K. M. Shahani et M. C. Badami. A resazurin disk assay method for detecting antibiotics and natural starter inhibitory activity in milk. J. Dairy Sei., 41, 1510. 1958.

[18] G. L. Sulverman and F. V. Kosikow ski. Systematic testing of inhibitory substances in milk. J. Milk and Food Technol., 15, 120. 1952.

[19] J. G. Vincent and H. W. Vincent. Filter paper disc modification of the Oxford cup penicillin determination. Proc. Soc. Exp. Biol. Med., $55,162,1944$.

[20] L. D. Witter and S. L. Tuckex. Variable factors in the new test for penicillin in milk. J. Milk and Food Technol., 23, 230. 1960.

[21] A. G. WoLIN and F. V. Kosikowskr. Formation of bacterial inhibitory zones in whey agar by raw milk. J. Dairy Sci., 41, 34. 1958.

\section{ÉTUdE ANALYTIQUE DES VARIATIONS DE QUALITÉ DES FROMAGES}

par

Dj. Filipovitch et R. Belanovitch (Belgrade)

De l'étude critique de l'un de nous sur les défauts de l'application pratique de différentes réglementations concernant le contrôle de la composition chimique des fromages (1) dans les divers pays, on peut voir combien il est difficile de réaliser le contrôle officiel de la qualité des fromages. Le problème se complique davantage dans les cas où la production se trouve aux mains d'un grand nombre de petits producteurs qui sont aussi vendeurs directs sur les petits et les grands marchés.

Nous avons fait avec beaucoup de patience des observations pendant des années à ce sujet et étudié les problèmes de la production

(1) Le Lait, 1960, 40, 22, 